\title{
Editorial
}

\section{Natural Therapies for Cardiovascular Diseases}

\author{
Xiaochen Yang, ${ }^{1}$ Guoyan Yang, ${ }^{2}$ Giuseppe Caminiti, ${ }^{3}$ and Francesca Borrelli ${ }^{4}$ \\ ${ }^{1}$ Department of Cardiology \& Health Care, Guanganmen Hospital, China Academy of Chinese Medical Sciences, Beijing, China \\ ${ }^{2}$ University of Western Sydney, Sydney, NSW, Australia \\ ${ }^{3}$ San Raffaele IRCCS Pisana, Rome, Italy \\ ${ }^{4}$ Universita degli Studi di Napoli Federico II, Naples, Italy
}

Correspondence should be addressed to Xiaochen Yang; avill1988@126.com

Received 30 November 2016; Accepted 1 December 2016

Copyright (c) 2016 Xiaochen Yang et al. This is an open access article distributed under the Creative Commons Attribution License, which permits unrestricted use, distribution, and reproduction in any medium, provided the original work is properly cited.

The natural therapies are generally referred to as these complementary and alternative therapies that have specific biological activity to human, including aromatherapy, dietbased therapies, folk medicine, megavitamin therapy, neural therapy, phytotherapy, and herbal medicine. In recent years, the potential benefit of natural therapy in improving chronic diseases prognosis has drawn more and more attention, and the use of natural therapy by physicians and patients has also increased markedly. From several decades ago to now, cardiovascular diseases (CVDs) continue to be the leading cause of mortality all over the world. In spite of some major advances in modern western medicine for treating CVDs, from revascularization to western medical therapy, the complications related to these procedures and recurrent acute cardiovascular events still occur in patients, who often suffer from unfavorable quality of life. With the increasing awareness of self-care of patients, natural therapies become popular among people with CVDs all over the world for their unique advantages in preventing diseases and relieving symptoms, rehabilitation, and health care. A handful of these natural therapies have purported cardiovascular effects. However, the role of these therapies in CVDs still needs more clinical evidence and definite mechanism of actions. Therefore, we opened this special issue which included researches of safety of natural therapies, side effects, clinical trials, and practice that assessed the roles of natural therapies for CVDs.

In this special issue, original research papers and reviews from different areas including China, Australia, Italy, and Malaysia are presented. These papers are focused on the mechanism of action of natural medicine and the systematic review of natural therapy in treating CVDs. The benefit of Eurycoma longifolia (E. longifolia) Jack, which originates from Southeast Asia, showed that the vasodilatory effect of a partially purified subfraction of root extracts of this natural plant. These novel findings may be of particular benefit to individuals who experience side effects with or who are intolerant of or not responsive to the conventional treatments. A pharmacological research provided evidence to support the traditional use of the Danshen-Sanqi combination for vascular disease through their synergistic interactions on anti-inflammatory pathways, which were examined in lipopolysaccharide-induced nitric oxide, tumor necrosis factor, and monocyte chemoattractant protein-1 generation pathways. Another pharmacological research showed that two compounds (ethyl caffeate and labiatenic acid) were selected as most promising CYP11B2 inhibitors, which provided a new approach to discover novel CYP11B2 inhibitors from Chinese medicine. In addition, a falconoid compound in many Chinese herbs and formula, luteolin, protects $\mathrm{H} 9 \mathrm{C} 2$ cells from $\mathrm{H} 2 \mathrm{O} 2$-induced apoptosis, which could be mediated by regulating the Akt-P53/Mdm2 apoptotic pathway. A systematic review suggested that various MediterrAsian diet products or natural extracts may represent a potential intervention treatment to raise HDL-C in dyslipidemic subjects. Meanwhile, the potential benefit of tetramethylpyrazine, the active component isolated from Rhizoma Chuanxiong, on the treatment of CVDs was also reviewed.

From the papers of our special issue, it can be concluded that original researches on mechanism of actions play an important role in the modernization and internationalization 
of natural medicine. However, the problems of indefinite mechanism of actions and lacking of high quality evidence still exist in the researches of natural therapies. In the future, more practical evidence like high quality random controlled trail (RCT) should be further strengthened in future researches.

Xiaochen Yang
Guoyan Yang
Giuseppe Caminiti

Francesca Borrelli 

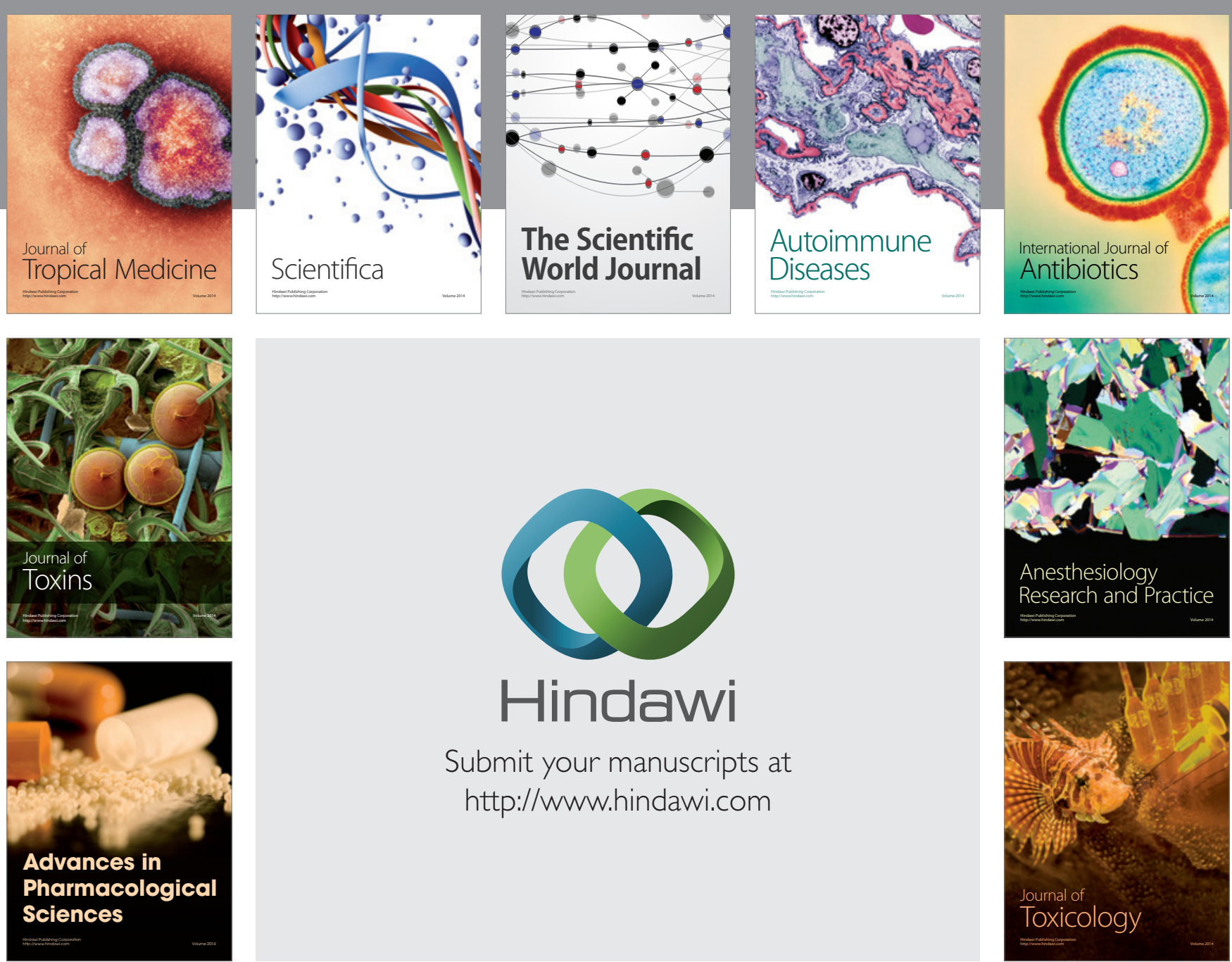

\section{Hindawi}

Submit your manuscripts at

http://www.hindawi.com
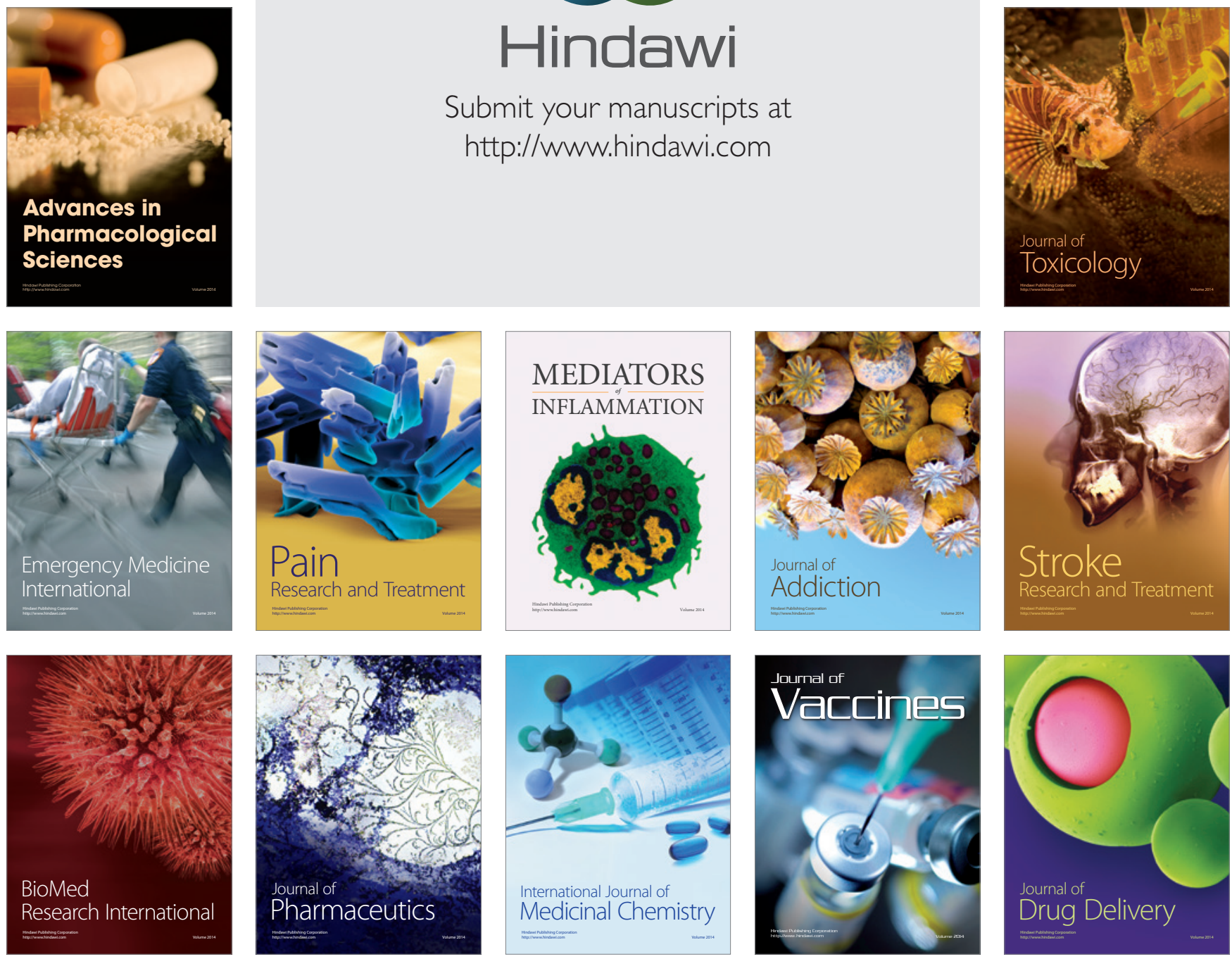